\title{
NON-REMOVABLE ARCH ORTHODONTIC APPLIANCES FOR TREATING CHILDREN WITH CONGENITAL MAXILLOFACIAL PATHOLOGIES - EFFICIENCY EVALUATION
}

\section{Vladimir Shkarin, Boris Davydov, Dmitry Domenyuk, Sergey Dmitrienko}

Congenital malformations are viewed as the most relevant urgent medical and social issues, which is the due to their high prevalence and the severity of morphological and functional changes in newborns. One of the varieties of the cleft alveolar process is a unilateral cleft where the jaw bones fragments are displaced, which is reflected in the dental arches linear parameters [1]. Numerous research methods have been proposed for diagnosis and the choice of treatment, where the leading one is the biometry of jaw stone models $[2,3,4,5,6]$. Treating children with congenital pathologies, as a rule, is complex and long-term. When treating children, primary teeth are fixed with non-removable elements shaped as thin-walled metal crowns, which impedes biometric examination and interpretation. Rationally explained expansion of the indications for jaw models biometric examination through the stages of orthodontic treatment would allow not only objective evaluation of the effectiveness of instrumental treatment aimed at optimizing the shape, dental arches dimensions, and occlusal connection, yet would also increase significantly the diagnostic value of morphometric study of dental arches in orthodontic practice, producing meaningful outcome for dentistry.

Arm. To identify the dynamics of change in the biometric parameters of the primary occlu-sion dental arches through the treatment of children with unilateral cleft alveolar process.

Materials and methods. A biometric study of dental arches was carried out on 37 children with unilateral cleft upper lip and alveolar process at the stage of the completely developed occlusion (4-6 years). The measurements were performed before and during the retention period of orthodontic treatment, with the edgewise technique being employed through the treatment. The prescription of the braces as well as the size of the metal arches selection was done in view of the primary occlusion specifics. The braces were soldered to metal thin-walled crowns, which were fixed on the primary teeth. After that, impressions were taken to obtain stone models, on which the linear parameters were measured. In the retention period, impressions were also obtained with the crowns to further carry out the biometrics, which eliminated any potential measurement errors since the measurements were carried out under the same conditions. At the biometric measurements of the jaw models, the intermolar distance was taken as the conventional midline of the upper jaw dental arch, from the middle of which a midline was drawn, which served as the reference for measuring the sagittal dimensions and allowed identifying the position of the interincisal point. At the same time, the diagonal dimensions of the dental arch were measured from the interincisal point to the canines and second primary molars.

Results ANid Discussion. The biometric measurement of the jaw stone models of the children with unilateral cleft alveolar process showed that after the treatment using non-removable orthodontic edgewise appliances there were significant changes in the major linear parameters of dental arches. The greatest changes were observed in the dental arches anterior section. On the intact side of the upper jaw dental arch, the difference in the transverse dimensions measured from the midline to the canines cusps was not significant $(p \geq 0.05)$ and was, before the treatment, $14.71 \pm 0.46$ $\mathrm{mm}$, while after the treatment it was $16.42 \pm 0.42 \mathrm{~mm}$. At the same time, at the cleft alveolar process side, the difference was significant $(p \leq 0.05)-11.49 \pm 0.79 \mathrm{~mm}$ and $16.36 \pm 0.51 \mathrm{~mm}$ before and after the treatment, respectively. In general, the width between the primary canines matched the age norm, since the arches dimensions used in the treatment were selected taking into account individual maxillofacial features. The changes in the anterior section revealed themselves in sagittal dimensions. The arch depth after the treatment increased significantly. There was a change in the interincisal point position observed, which coincided with the facial aesthetic center line. The dental arch depth was about $9.5 \mathrm{~mm}$ and there were no significant differences identified between the indices on each side. For the same reason, there was an increase in the overall dental arch depth and the diagonal dimensions on both sides. The dental arch depth on the intact side before the treatment was $23.72 \pm 0.84 \mathrm{~mm}$, while after the treatment it went up to $25.57 \pm 0.42 \mathrm{~mm}$. On the cleft side, the values were $25.57 \pm 0.42 \mathrm{~mm}$ and 25.44 $\pm 0.48 \mathrm{~mm}$, respectively. The greatest changes in the 
diagonal dimensions of the arch anterior sec-tion and the entire dental arch significantly increased on the side of the cleft maxillary alveolar process. Before the treatment, the frontal canine diagonal was $13.41 \pm$ $0.85 \mathrm{~mm}$, and after treat-ment it was $18.23 \pm 0.37 \mathrm{~mm}$. On the intact side, the values were $16.52 \pm 0.73 \mathrm{~mm}$ and $18.31 \pm 0.39 \mathrm{~mm}$, respectively. The front-molar diagonal on both sides was an average of $35.5 \mathrm{~mm}$ and matched the average age indices.

CONCLUSION. The obtained outcomes showed that after the treatment with non-removable orthodontic edgewise technique, there were certain changes in the basic parameters of the dental arches in children during their primary teeth occlusion, which complied with the age norm. The use of non-removable orthodontic arch appliances is an effective treatment for children with congenital maxillofacial pathologies.

\section{REFERENCES}

1. Domenyuk D.A., Vedeshina E.G., Dmitrienko S.V. Dynamics of changes in transverse and frontalretromolar jaw sizes in children throughout the orthodontic treatment stages. Kubanskij nauchnyj medicinskij vestnik. 2016; (3):51-59. (In Russ.) DOI:10.25207/1608-6228-2016-3-51-59.
2. Domenyuk D.A., ShKarin V.V., PorfiRiadis M.P., DMitrienko D.S., DMITRIENKo S.V. Algorithm for forecasting the shape and size of dental arches front part in case of their deformations and anomalies. Archiv EuroMedica, 2017; 7(2): 105-110.

3. Domenyuk, D.A. Correlation of dental arch major linear parameters and odontometric indices given physiological occlusion of permanent teeth in various face types / D.A. Domenyuk, E.G. Vedeshina, S.V. Dmitrienko // Archiv EuroMedica. - 2016. - Vol. 6. № 2. - P. 18-22.

4. Domenyuk D.A., Shkarin V.V., Porfiriadis M.P., DMitrienKo D.S., DMITRIENKo S.V. Setting reference points for key teeth location in cases of abnormal dental arch shape. Archiv EuroMedica, 2017; 7(2): 111-117.

5. Domenyuk, D.A. Mistakes in Pont (Linder-Harth) method used for diagnosing abnormal dental arches in transversal plane / D.A. Domenyuk, E.G. Vedeshina, S.V. Dmitrienko // Archiv EuroMedica. - 2016. - Vol. 6. - № 2. - P. 23-26.

6. Shkarin V.V., Domenyuk D.A., Porfiriadis M.P., DMitrienko D.S., DMitrienko S.V. Mathematical and graphics simulation for individual shape of maxillary dental arch // Archiv Euro-Medica, 2017. - Vol. 7. - № 1. - P. 60-65. 